\title{
Efecto del tratamiento térmico sobre las propiedades mecánicas y microestructura de un acero para tubería API 5CT J55
}

\author{
Effect of Heat Treatment on the Mechanical Properties \\ and Microstructure of a API 5CT J55 Pipeline Steel
}

\author{
Soria-Aguilar Ma. de Jesús \\ Universidad Autónoma de México \\ Facultad de Metalurgia \\ Correo:mjsoriaa@yahoo.com.mx \\ Reyes-Guzmán Facundo \\ Altos Hornos de México, S.A.B. de C.V. \\ Correo:freyesguzman@gan.com.mx \\ Carrillo-Pedroza Francisco Raúl \\ Universidad Autónoma de México \\ Facultad de Metalurgia \\ Correo:frrcarrillo@yahoo.com.mx
}

\author{
García-Garza Fernando \\ Altos Hornos de México, S.A.B. de C.V. \\ Correo:fgarcia@gan.com.mx \\ Álvarez-Jiménez Humberto \\ Altos Hornos de México, S.A.B. de C.V. \\ Correo:halvarezjimenez@gan.com.mx \\ Silva-Guajardo Luis Antonio \\ Altos Hornos de México, S.A.B. de C.V. \\ Correo:lasilvag@gan.com.mx
}

Información del artículo: recibido: mayo de 2014, aceptado: marzo de 2015

\section{Resumen}

En el presente trabajo se investigó el efecto de dos ciclos de tratamientos térmicos sobre la microestructura y propiedades mecánicas de un acero soldado API 5CT J55. Las pruebas se realizaron basándose en un diseño experimental tipo Taguchi. El impacto de los parámetros del tratamiento térmico sobre la microestructura y las propiedades mecánicas se determinó mediante un análisis estadístico de varianza (ANOVA) del arreglo Taguchi ortogonal (L9). De los resultados del ANOVA se obtuvieron ecuaciones empíricas para optimizar las condiciones de tratamiento térmico, de las cuales se pueden lograr las mejores propiedades mecánicas.

\section{Descriptores:}

- acero

- ANOVA

- API

- microestructura

- tratamiento térmico

- propiedades 


\begin{abstract}
The effects of two different post-weld heat treatment cycles on the microstructure and mechanical properties of welded API 5CT J55 steels were investigated in the present work. Experiments were carried out based on a Taguchi experimental design. Ortogonal arrays (L9) of Taguchi and statistical analysis of variance (ANO$V A$ ) were employed to determine the impact of the heat treatment parameters on the microstructure and mechanical properties of experimental steel. From the results of ANOVA, there were obtained the empirical equations for optimizing the heat treating conditions that lead to the best mechanical properties.
\end{abstract}

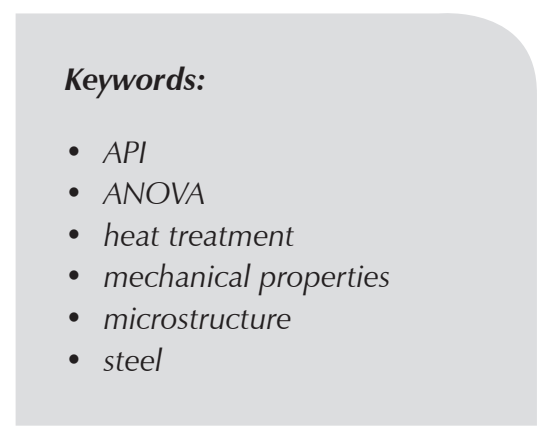

\section{Introducción}

La demanda de energía limpia y las necesidades de transporte con mayores volúmenes de gas natural a través de tuberías de acero de alta presión, han dado lugar a la fabricación de aceros de alta resistencia para tuberías modernas. La combinación de la tecnología de laminación de enfriamiento acelerada y controlada se utilizan para producir aceros de baja aleación y alta resistencia (HSLA) (Hwang et al., 2005). Estos aceros se utilizan para tubos de gran diámetro en redes de gasoductos con especificaciones basadas en los estándares según los códigos API (American Petroleum Institute) (API Specifications, 2004). Estos aceros deben cumplir con otros requisitos o características propias de su aplicación, tales como soldabilidad, formabilidad, tenacidad a la fractura, ductilidad a la temperatura de transición frágil, resistencia a la fisuración inducida por hidrógeno, resistencia a la corrosión bajo tensión y la resistencia a la fatiga (Hwang et al., 2005, Kishor y Kolhe, 2008). Estas propiedades se afectan principalmente por la microestructura, la cual a su vez, depende en gran medida de micro-elementos de aleación, tales como Mn, Nb, V, Ti, Mo y Ni. Por ejemplo, el manganeso y el molibdeno se añaden a la aleación para promover la formación de la microestructura acicular en la transformación de la austenita (Junhua et al., 2004). Titanio y niobio se utilizan para lograr una microestructura fina de austenita durante la laminación, que a su vez se transforma en una microestructura de ferrita acicular en el enfriamiento acelerado.

Para obtener una microestructura adecuada son importantes los tratamientos térmicos y, en especial, aquellos dados al acero para tubería que se soldan, conocidos como tratamiento térmico posterior a la soldadura (PWHT). Las funciones de un PWHT son revenir la martensita en el metal de soldadura y zona afectada por el calor, con el fin de reducir la dureza, aumentar la tenacidad y disminuir las tensiones residuales asociadas con la soldadura (Kumar et al., 2010; Hashemi y Mohammadyani, 2012).

Otro aspecto de los PWHT es la relevación de esfuerzos, con el objetivo de reducir las tensiones que permanecen encerradas en una estructura como consecuencia de los procesos de fabricación. Hay muchas fuentes de tensiones residuales, y las que se deben a la soldadura, son de una magnitud aproximada a la resistencia y a la fluencia del material base. Generalmente, el tratamiento térmico de relevado, llamado también de revenido, consiste en un calentamiento uniforme de la estructura a una temperatura suficientemente alta, pero por debajo de la temperatura de transformación austenítica, seguido de un enfriamiento uniforme. La temperatura que se alcanza durante el tratamiento, tiene un efecto mucho mayor en el relevado de tensiones que la cantidad de tiempo que se mantiene la muestra a esa temperatura (Armentani et al., 2007). Cuanto más cerca esté a la temperatura crítica de re-cristalización, será más eficaz la eliminación de las tensiones residuales. Algunos estudios concluyen que la microestructura de la zona caliente durante la soldadura es responsable del deterioro de la propiedad de esta y la susceptibilidad de agrietamiento en frío. Dichos estudios también indican que el aumento de precalentamiento y PWHT incrementa el tamaño de grano en las microestructuras de soldadura, influenciando significativamente las propiedades de las uniones soldadas (Barsoum, 2009; Francis et al., 2009).

En los aceros para tubería, y en particular, en aceros bajo carbono, se opta por una microestructura de grano fino $(<2 \mu \mathrm{m})$, ya que esta proporciona mayor resistencia y tenacidad a la fractura. Por lo tanto, el refinamiento de tamaño de grano puede representar una de las vías más prometedoras para mejorar las 
propiedades mecánicas (Santos et al., 2003). En el caso del PWHT, es importante determinar los parámetros del ciclo de tratamientos térmicos. La normalización y tratamiento de austenizado y revenido generalmente se lleva a temperaturas mayores del punto de transformación $\mathrm{A}_{\mathrm{C} 3}$. Los ciclos de calentamiento, incluyendo las velocidades de calentamiento, las tasas de enfriamiento y tiempos de retención deben calcularse en función de las velocidades de línea y la longitud de la soldadura (Majta y Muszka, 2007). Algunos investigadores han observado que las fases duras, tales como martensita y bainita se presentan en las microestructuras del material soldado, encontrando que se forma preferencialmente martensita y una alta densidad de dislocaciones tanto en aceros microaleados (grado X65) como en aceros con microestructura de grano fino (Chung et al., 2012). Estos autores observaron que las microestructuras presentes en la línea de unión de muestras normalizadas constan de ferrita poligonal y una pequeña cantidad de perlita. Sin embargo, las microestructuras después del temple en agua consistían en su mayoría del tipo martensita. Aunque los valores de tenacidad mejoraron tras el templado para los aceros de grado X65 y de grano fino, fue difícil determinar una diferencia significativa entre las microestructuras de las muestras de austenizado y revenido.

De acuerdo con lo anterior, la determinación de los parámetros óptimos para los tratamientos térmicos conlleva la realización de muchos experimentos que, sin la ayuda de un buen diseño experimental, puede volverse complicado. En este sentido, los métodos estadísticos de diseño de experimentos y, en particular, el diseño ortogonal Taguchi, ofrecen la ventaja de estudiar el efecto de varios parámetros con un número pequeño de experimentos. Este tipo de diseños se ha empleado en la optimización de diferentes procesos de manufactura, permitiendo la determinación rápida de los factores principales y su efectos en un proceso, además de los ahorros en tiempos y costos (Saini et al., 2013; Noor et al., 2014; Tutar et al., 2014).

Por lo tanto, el principal objetivo de este trabajo fue estudiar el efecto de los tratamientos de austenizado y revenido en la evolución microestructural, las propiedades mecánicas de un acero API 5CT J55, empleando un diseño ortogonal Taguchi L9 y el análisis de varianza (ANOVA) para generar ecuaciones empíricas que permitan la optimización de las condiciones de tratamiento térmico para mejorar las propiedades mecánicas de este grado de acero.

\section{Desarrollo exprimental}

\section{Materiales de ensayo}

Los materiales utilizados para el desarrollo de las pruebas experimentales fueron probetas de acero API 5CT J55 con dimensiones $45.7 \mathrm{~cm}$ de longitud, $0.4826 \mathrm{~cm}$ de espesor y $3.55 \mathrm{~cm}$ de ancho. Las muestras se obtuvieron de tubo conformado y soldado. Las muestras se procesaron por tratamiento térmico y ensayos físicos, tal como se describe a continuación.

\section{Tratamientos térmicos}

Se realizaron diferentes tipos de tratamientos térmicos en las probetas previamente seleccionadas con ayuda de una mufla marca BARNSTEAD/THERMOLYNE modelo CP536LS con controlador de precisión de $\pm 5^{\circ} \mathrm{C}$, a temperaturas de austenizado de 890,920 y $950^{\circ} \mathrm{C} \mathrm{du}$ rante 22, 24 y 26 minutos, seguido de un enfriamiento en agua. Posterior al enfriamiento, las probetas se trataron térmicamente por revenido a 460,480 y $500^{\circ} \mathrm{C}$ durante 22,24 y 26 minutos, enfriadas al aire, a fin de relevar los esfuerzos internos retenidos en la microestructura debido al temple. Los tiempos del tratamiento térmico de austenizado se calcularon a partir de las especificaciones establecidas por la norma API 5CT (considerando el espesor y composición química del acero). La temperatura de tratamiento térmico de austenizado se calculó con ayuda de la ecuación de Andrews (1965) tomando en cuenta la composición química correspondiente al grado de acero. Las temperaturas de tratamiento térmico de revenido se establecieron de acuerdo con las pruebas preliminares y se realizaron por duplicado siguiendo el diseño experimental Taguchi L9 mostrado en la tabla 1.

\section{Ensayos físicos}

Las muestras obtenidas, antes y después de los tratamientos térmicos, se sometieron a diferentes pruebas físicas, las cuales se describen a continuación:

\section{Ensayo de dureza}

La determinación de la dureza se realizó en un durómetro marca Wilson modelo INSTRON en la escala Rockwell C (HRC) de acuerdo con la norma ASTM E-18. En el ensayo se utilizó un indentador cónico con punta diamante. Para realizar la medición, la pieza se precargó con $10 \mathrm{~kg}$ y posteriormente se aplicó una carga de 150 $\mathrm{kg}$. Una vez que se realizó el ensayo, el equipo emplea- 
Tabla 1. Diseño experimental y condiciones de las pruebas de tratamiento térmico para muestras de tubo

\begin{tabular}{ccccc}
\hline \multicolumn{5}{c}{ Factores } \\
\hline Prueba & $\begin{array}{c}\text { Temperatura austenizado } \\
\left({ }^{\circ} \mathrm{C}\right)\end{array}$ & $\begin{array}{c}\text { Tiempo austenizado } \\
\text { (minutos) }\end{array}$ & $\begin{array}{c}\text { Temperatura } \\
\text { revenido }\left({ }^{\circ} \mathrm{C}\right)\end{array}$ & $\begin{array}{c}\text { Tiempo revenido } \\
(\text { minutos })\end{array}$ \\
\hline 2 & 890 & 22 & 460 & 22 \\
3 & 890 & 24 & 480 & 24 \\
4 & 890 & 26 & 500 & 26 \\
5 & 920 & 22 & 480 & 26 \\
6 & 920 & 24 & 500 & 22 \\
7 & 920 & 26 & 460 & 24 \\
8 & 950 & 22 & 500 & 26 \\
9 & 950 & 24 & 460 & 22 \\
\hline
\end{tabular}

do mostró directamente la lectura del valor de la dureza resultante. La probeta se ensayó en tres ocasiones, lo que marcó un promedio con base en la norma.

\section{Ensayo de tensión}

Los ensayos de tensión se realizaron en una máquina universal de 300,000 lb (15 kN) de carga, marca TINIUS OLSEN Modelo Súper L 300/602, de acuerdo con el procedimiento de la norma ASTM A370, con la finalidad de determinar el porcentaje de elongación y los valores de resistencia a la tensión (último esfuerzo a la tensión y límite elástico).

\section{Ensayo metalográfico}

La microestructura del acero 5CT J55 se caracterizó empleando técnicas de microscopia óptica y microscopia electrónica de barrido. Para tal efecto, algunas de las muestras ensayadas se seleccionaron de acuerdo con los resultados obtenidos de las pruebas de dureza y ensayo a la tensión. Dichas muestras se cortaron con ayuda de una sierra vertical Modelo BIRMINGHAM, serie $\mathrm{Kv}-50 \mathrm{a}$ y se montaron en baquelita (resina), usando una montadora en caliente marca STRUERS modelo LABOPRESS-3, con capacidad de fuerza de 30 $\mathrm{KN}$, posteriormente se obtuvo una pastilla de $40 \mathrm{~mm}$. Estas muestras fueron desbastadas y luego pulidas con alúmina hasta obtener un acabado espejo. Posteriormente, las probetas fueron atacadas por la técnica de inmersión en una solución de nítal a 3\% por un tiempo no mayor a 5 segundos, para observarse después en un microscopio marca OLIMPUS Modelo PMG3 con la finalidad de observar la microestructura resultante, de conformidad a la norma ASTM E-45, así como determinar el porcentaje de cada fase y el tamaño de grano. Algunas muestras se observaron en un microscopio electrónico de barrido (marca JEOL modelo JSM-IT300LV).

\section{Análisis y discusión de resultados}

Los datos obtenidos de los ensayos físicos de las muestras tratadas térmicamente se analizaron estadísticamente para determinar la variable respuesta de cada propiedad mecánica del material mediante un análisis de varianza (ANOVA) y la obtención de curvas unifactoriales. Las curvas paramétricas se obtuvieron de un modelo de regresión múltiple lineal, a fin de visualizar los efectos significativos más importantes en cuanto a las variables que afectan las propiedades físicas del acero API 5CT J55.

Para determinar el efecto de las variables de temperatura y tiempos de tratamientos de austenizado y revenido, se llevaron a cabo una serie de pruebas de acuerdo con el diseño Taguchi L9 (tabla 2). Esta tabla muestra los valores obtenidos de los ensayos de tensión y dureza de las probetas ensayadas provenientes de tubo.

De acuerdo con los resultados, las probetas ensayadas cumplen con la especificación a la norma en relación a límite elástico y último esfuerzo a la tensión, a excepción de la probeta 1 (muestra de tubo sin ningún tratamiento) y la probeta 8 .

Para el análisis de los datos resultantes se utilizaron los métodos estadísticos más comunes en los diseños ortogonales tipo Taguchi: el análisis de varianza (ANOVA) de los datos, los gráficos de la curva de la respuesta promedio y, adicionalmente, una regresión lineal múltiple (Burgman, 1985; Ross, 1996). A continuación se muestra una breve descripción de estos modelos.

La curva de la respuesta promedio grafica el promedio obtenido de los datos respuesta de cada experimento realizado a un cierto nivel de un parámetro, indicando una tendencia del efecto del mismo en el resultado. Es una representación gráfica del efecto de cada parámetro sobre la respuesta. Este tipo de gráfico permite visualizar fácilmente cómo se afecta la respuesta (resultado) por los niveles para cada uno de los parámetros o factores experimentales. 
El ANOVA, por su parte, identifica el factor más significativo. Este análisis se obtiene comparando los diversos valores medios para determinar si alguno de ellos difiere significativamente del resto, es decir, estimando la varianza entre las respuestas experimentales de los diferentes niveles para cada factor. La varianza es la media de la suma de los cuadrados, la cual se estima para cada parámetro o tratamiento. La suma de cuadrados mide la diferencia entre los resultados individuales (niveles) de un factor, mediante la siguiente expresión

$S S=\sum_{i=1}^{i}\left(y_{i}-\bar{y}\right)^{2}$

Donde

SS = suma de cuadrados

$i \quad$ número de niveles

$y_{i}=$ respuesta o dato para cada nivel $i$ de un factor

$\bar{y}=$ promedio de las respuestas de un factor
De acuerdo con el diseño de experimentos se tienen 3 niveles para cada factor, el grado de libertad para cada factor es (3-1) igual a 2. Así, la media de la suma de cuadrados o varianza se obtiene al dividir la suma de cuadrados (ecuación 1) entre los grados de libertad. De esta manera, cuando un factor exhibe un valor de varianza proporcionalmente más grande que los demás factores, es indicativo de que dicho factor tiene mayor impacto o es más significativo sobre la respuesta.

Finalmente, la regresión lineal múltiple expresa una respuesta en función de las variables explicativas (factores), a través de un modelo cuyos valores de los parámetros para cada factor son la variación media que experimenta la variable respuesta cuando el valor del factor cambia en una unidad. Por lo anterior, se describen y analizan los resultados obtenidos.

Los resultados del ANOVA se muestran en la tablas 3, 4 y 5 para las respuestas dadas en las propiedades de límite elástico (LE), último esfuerzo a la tensión (UT) y dureza después del revenido, respectivamente. La tabla 6 muestra las respuestas obtenidas de la relación UT/LE.

Tabla 2. Valores obtenidos de ensayos de tensión y dureza en muestras de tubo

\begin{tabular}{|c|c|c|c|c|c|c|}
\hline Probeta & $\begin{array}{c}\text { Límite elástico } \\
(\mathrm{MPa})\end{array}$ & $\begin{array}{l}\text { Última tensión } \\
(\mathrm{MPa})\end{array}$ & Elongación (\%) & $\begin{array}{l}\text { Relación } \\
\text { L.E/U.T }\end{array}$ & $\begin{array}{c}\text { Dureza austenizado } \\
\text { (HRC) }\end{array}$ & Dureza revenido (HRC) \\
\hline 1 & 483.91 & 555.71 & 26.7 & 0.87 & & \\
\hline 2 & 962.33 & 990.44 & 13.0 & 0.97 & 40.2 & 39.4 \\
\hline 3 & 859.00 & 906.22 & $\mathrm{f} / \mathrm{p}$ & 0.95 & 42.7 & 33.8 \\
\hline 5 & 862.09 & 893.46 & $\mathrm{f} / \mathrm{p}$ & 0.96 & 46.3 & 35.8 \\
\hline 6 & 806.44 & 867.81 & 14.7 & 0.93 & 43.8 & 38.7 \\
\hline 7 & 897.79 & 959.16 & $\mathrm{f} / \mathrm{p}$ & 0.94 & 40.8 & 40.2 \\
\hline 8 & 743.96 & 820.15 & 14.4 & 0.91 & 44.6 & 38.17 \\
\hline 9 & 853.03 & 917.75 & $\mathrm{f} / \mathrm{p}$ & 0.93 & 42.3 & 41.3 \\
\hline 10 & 804.60 & 885.06 & $\mathrm{f} / \mathrm{p}$ & 0.91 & 41.6 & 38.8 \\
\hline $\begin{array}{c}\text { Especificación } \\
5 \text { CT J55 }\end{array}$ & 758-965 Мра & $\begin{array}{l}863 \mathrm{Mpa} \\
\text { mínimo }\end{array}$ & 15\% mínimo & $\mathrm{n} / \mathrm{a}$ & & \\
\hline
\end{tabular}

Tabla 3. Análisis de varianza (ANOVA) para límite elástico (LE)

\begin{tabular}{lccc}
\hline \multicolumn{1}{c}{ Factor } & $\begin{array}{c}\text { Grados de } \\
\text { libertad }\end{array}$ & $\begin{array}{c}\text { Suma de } \\
\text { cuadrados }\end{array}$ & $\begin{array}{c}\text { Media de la suma } \\
\text { de cuadrados }\end{array}$ \\
\hline Temp. Austenizado & 2 & 191.81 & 95.91 \\
Tiempo Austenizado & 2 & 14.33 & 7.16 \\
Temp. Revenido & 2 & 445.52 & 222.76 \\
Tiempo Revenido & 2 & 19.61 & 9.81 \\
Total (Ajustado) & 8 & 671.28 & \\
Total & 9 & & \\
\hline
\end{tabular}




\begin{tabular}{lccc}
\multicolumn{4}{c}{ Tabla 4. Análisis de varianza (ANOVA) para último esfuerzo a la tensión (UT) } \\
\hline \multicolumn{1}{c}{ Factor } & $\begin{array}{c}\text { Grados de } \\
\text { libertad }\end{array}$ & $\begin{array}{c}\text { Suma de } \\
\text { cuadrados }\end{array}$ & $\begin{array}{c}\text { Media de la suma } \\
\text { de cuadrados }\end{array}$ \\
\hline Temp. Austenizado & 2 & 73.54 & 36.77 \\
Tiempo Austenizado & 2 & 1.46 & 0.73 \\
Temp. Revenido & 2 & 343.75 & 171.87 \\
Tiempo Revenido & 2 & 17.57 & 8.78 \\
Total (Ajustado) & 8 & 436.31 & \\
Total & 9 & & \\
\hline
\end{tabular}

\begin{tabular}{lccc} 
Tabla 5. Análisis de varianza (ANOVA) para Dureza & \\
\hline \multicolumn{1}{c}{ Factor } & $\begin{array}{c}\text { Grados de } \\
\text { libertad }\end{array}$ & $\begin{array}{c}\text { Suma de } \\
\text { cuadrados }\end{array}$ & $\begin{array}{c}\text { Media de la suma } \\
\text { de cuadrados }\end{array}$ \\
\hline Temp. Austenizado & 2 & 6.795755 & 3.397878 \\
Tiempo Austenizado & 2 & 3.79 & 1.90 \\
Temp. Revenido & 2 & 26.23 & 13.11 \\
Tiempo Revenido & 2 & 4.08 & 2.04 \\
Total (Ajustado) & 8 & 40.90 & \\
Total & 9 & &
\end{tabular}

Tabla 6. Análisis de varianza (ANOVA) para UT/LE

\begin{tabular}{lccc}
\hline \multicolumn{1}{c}{ Factor } & $\begin{array}{c}\text { Grados de } \\
\text { libertad }\end{array}$ & $\begin{array}{c}\text { Suma de } \\
\text { cuadrados }\end{array}$ & $\begin{array}{c}\text { Media de la suma } \\
\text { de cuadrados }\end{array}$ \\
\hline Temp. Austenizado & 2 & 0.00186 & 0.00093 \\
Tiempo Austenizado & 2 & 0.00060 & 0.00030 \\
Temp. Revenido & 2 & 0.00087 & 0.00043 \\
Tiempo Revenido & 2 & 0.00007 & 0.00003 \\
Total (Ajustado) & 8 & 0.00340 & \\
Total & 9 & & \\
\hline
\end{tabular}

Los resultados del ANOVA indican que la temperatura de revenido es el factor más significativo por el mayor valor en la media de la suma de cuadrados (el valor de la media es indicativo de que los niveles de un factor producen mayor variabilidad en la respuesta). La temperatura de revenido tiene mayor efecto en los resultados de límite elástico (tabla 3), de último esfuerzo a la tensión (tabla 4) y en la dureza (tabla 5). Posteriormente siguen como factores significativos, la temperatura de austenizado y finalmente los tiempos de austenizado y revenido, los cuales muestran un menor valor de la media por la suma de cuadrados con respecto al valor de la media de la temperatura de revenido. Sin embargo, es importante notar en la tabla 6, la cual muestra la relación UT/LE, que el factor con mayor media es la temperatura de austenizado. Este resultado se discute más adelante.
El resultado del ANOVA se presenta de manera gráfica en las figuras 1 y 2, mediante las curvas unifactoriales de la respuesta promedio, la cual presenta el efecto de cada nivel para cada factor sobre las respuestas. En la figura 1 se puede observar claramente el efecto del factor o variable por nivel en la respuesta. En cuanto a la temperatura de revenido, la figura muestra el impacto de esta variable, la cual, al aumentar la temperatura de 460 a $500^{\circ} \mathrm{C}$, disminuyen tanto LE como UT de una manera significativa y casi linealmente, decreciendo ambas propiedades en casi $14 \mathrm{y}$ $11 \%$, respectivamente, asimismo, pasando del valor límite de especificación de 125 unidades (862 MPa) para UT después de los $480^{\circ} \mathrm{C}$. Con respecto a la temperatura de austenizado, el efecto es menor al cambiar las temperaturas entre 890,920 y $950^{\circ} \mathrm{C}$, observándose un decremento tanto en LE como en UT de 8 y $5 \%$, 
respectivamente. Finalmente, las gráficas confirman el resultado del ANOVA, observándose que tanto el tiempo de austenizado como el de revenido no afectan significativamente los valores de LE y UT.

En el caso de la relación UT/LE y Dureza, mostrados en la figura 2, los resultados muestran que para el caso de la relación UT/LE se mantiene la tendencia de los parámetros individuales (UT y LE), lo que indica que a menor temperatura de revenido y temperatura de austenizado, se obtienen los mayores valores de dicha relación. Al aumentar ambas temperaturas, los valores decrecen de manera importante, por debajo de la relación $\mathrm{UT} / \mathrm{LE}=0.94$ a temperaturas de revenido y austenizado menores de 480 y $920^{\circ} \mathrm{C}$, respectivamente. En el caso de la dureza, el comportamiento es inverso, es decir, a mayor temperatura de austenizado y menor temperatura de revenido, se tiene mayor dureza, con diferencias entre 5 y $10 \%$, respectivamente.

Con base en los resultados del ANOVA y analizando los datos mediante Regresión Lineal Múltiple, se obtuvieron las siguientes relaciones empíricas para cada una de las propiedades físicas del acero en tubo:

Límite elástico, LE (MPa)

$3808.76-1.368\left(\mathrm{~T}_{\text {austenizado }}\right)-03.174\left(\mathrm{t}_{\text {austenizado }}\right)-$

$3.139\left(\mathrm{~T}_{\text {revenido }}\right)-5.178\left(\mathrm{t}_{\text {revenido }}\right)$

Esfuerzo último a la tensión, UT (MPa)

$3025.21-0.702\left(\mathrm{~T}_{\text {austenizado }}\right)-0.597\left(\mathrm{t}_{\text {austenizado }}\right)-$

$2.722\left(\mathrm{~T}_{\text {revenido }}\right)-6.619\left(\mathrm{t}_{\text {revenido }}\right)$

Relación UT/LE

$1.8-0.00061\left(\mathrm{~T}_{\text {austenizado }}\right)-0.00416\left(\mathrm{t}_{\text {austenizado }}\right)-$ $0.0005\left(\mathrm{~T}_{\text {revenido }}\right)+0.0016\left(\mathrm{t}_{\text {revenido }}\right)$

Dureza (HRC)

$51.656+0.00619\left(\mathrm{~T}_{\text {austenizado }}\right)+0.0 .0458\left(\mathrm{t}_{\text {austenizado }}\right)-$

$0.058\left(\mathrm{~T}_{\text {revenido }}\right)+0.359\left(\mathrm{t}_{\text {revenido }}\right)$

Donde

$\mathrm{T}_{\text {austenizado }}=$ Temperatura de austenizado, ${ }^{\circ} \mathrm{C}$

$t_{\text {austenizado }}=$ Tiempo de austenizado, minutos

$\mathrm{T}_{\text {revenido }}=$ Temperatura de revenido, ${ }^{\circ} \mathrm{C}$

$t_{\text {revenido }}=$ Tiempo de revenido, minutos

Con estas ecuaciones es posible obtener curvas paramétricas, como las que se muestran en la figura 3, que son una herramienta visual poderosa para encontrar de manera fácil y rápida las condiciones de temperatura de revenido y temperatura de austenizado (factores principales), con las cuales se pueden obtener o predecir las propiedades de límite elástico (LE) y último esfuerzo a la tensión (UT), así como la relación entre ambos (UT/ LE) y la Dureza, en el rango de valores estudiados. En estas curvas parámetricas se confirma nuevamente que el parámetro que más afecta las propiedades físicas es la temperatura de revenido. Solo en la relación UT/LE se puede apreciar mejor el efecto de la temperatura de austenizado. Esto es importante, ya que permite identificar con mayor claridad aquellos valores de temperaturas que maximicen las propiedades físicas. En este caso, a partir de dichas curvas, es posible confirmar que los valores de propiedades mecánicas más altas se obtienen a las menores temperaturas de austenizado y revenido experimentadas en este trabajo. La razón de este comportamiento en las propiedades mecánicas se puede discutir analizando los cambios microestructurales que ocurren durante los tratamientos térmicos. Para ello, se seleccionaron algunas muestras para su analisis microestructural, de acuerdo con los resultados obtenidos de las pruebas mecánicas realizadas a las probetas con tratamiento termico de austenizado y revenido.

La figura 4 muestra una comparación de las fotomicrografías obtenidas de las probetas procedentes del tubo, las cuales se ensayaron bajo los tratamientos experimentales 3 y 7 . Las muestras se seleccionaron a partir de los resultados obtenidos en los ensayos de tensión. En la figura 4, la fotomicrografía (a) corresponde a la probeta tratada térmicamente por austenizado a la temperatura de $890^{\circ} \mathrm{C}$ por 24 minutos y templada, y la fotomicrografía (b), correspondiente a la probeta tratada bajo las mismas condiciones de austenizado y luego revenida a $480^{\circ} \mathrm{C}$ por 24 minutos fue enfriada al aire. En el caso de las fotomicrografías (c) y (d), corresponden a la muestra tratada por austenizado $\left(920^{\circ} \mathrm{C}\right.$ por 26 minutos) y austenizada (mismas condiciones) y revenida $\left(460^{\circ} \mathrm{C}\right.$ por 24 minutos), respectivamente. Como se puede observar en esta figura, las probetas tratadas por austenizado seguido del temple en agua (a) y (c) presentan una microestructura compuesta principalmente de agujas de martensita con regiones de austenita retenida. La microestructura es típica para este tipo de aceros, donde el temple permite la transformación de la mayor parte de austenita a martensita, que incrementa notablemente las propiedades mecánicas con respecto a la probeta sin tratamiento (Probeta 1 de la tabla 2).

Por otro lado, las figuras $4 \mathrm{~b}$ y d, fotomicrografías correspondientes a las probetas tratadas por austenizado y revenido, presentan martensita revenida y algunas regiones de austenita, observándose que, tal como lo 

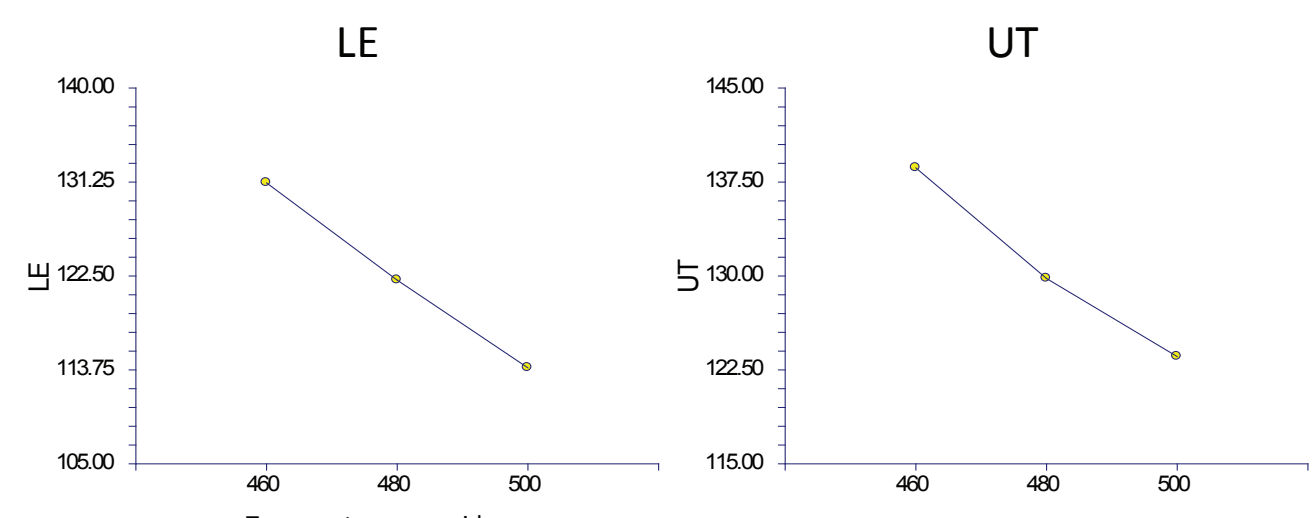

Temperatura revenido

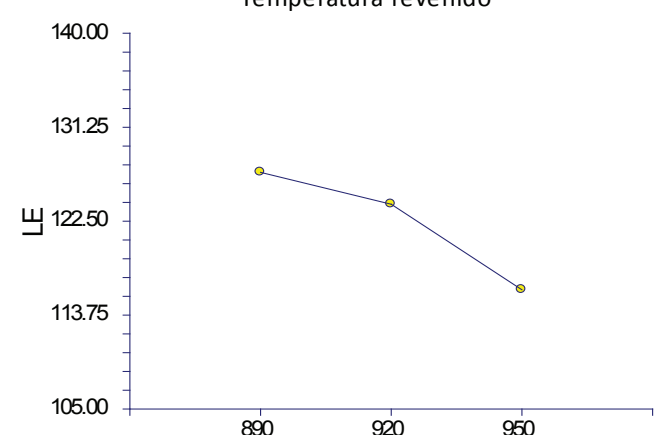

Temperatura austenizado
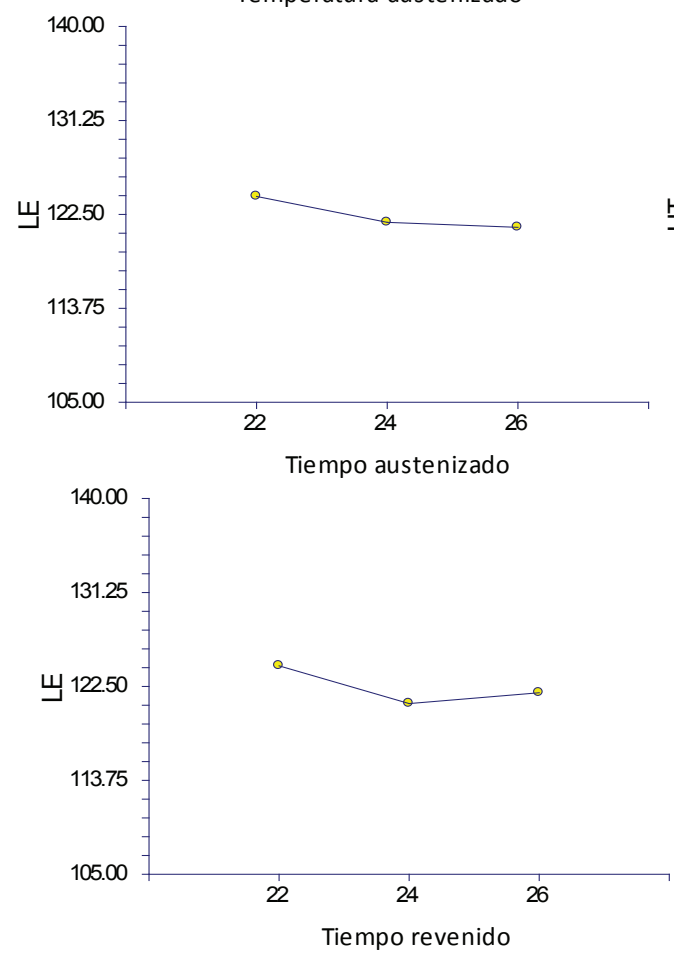
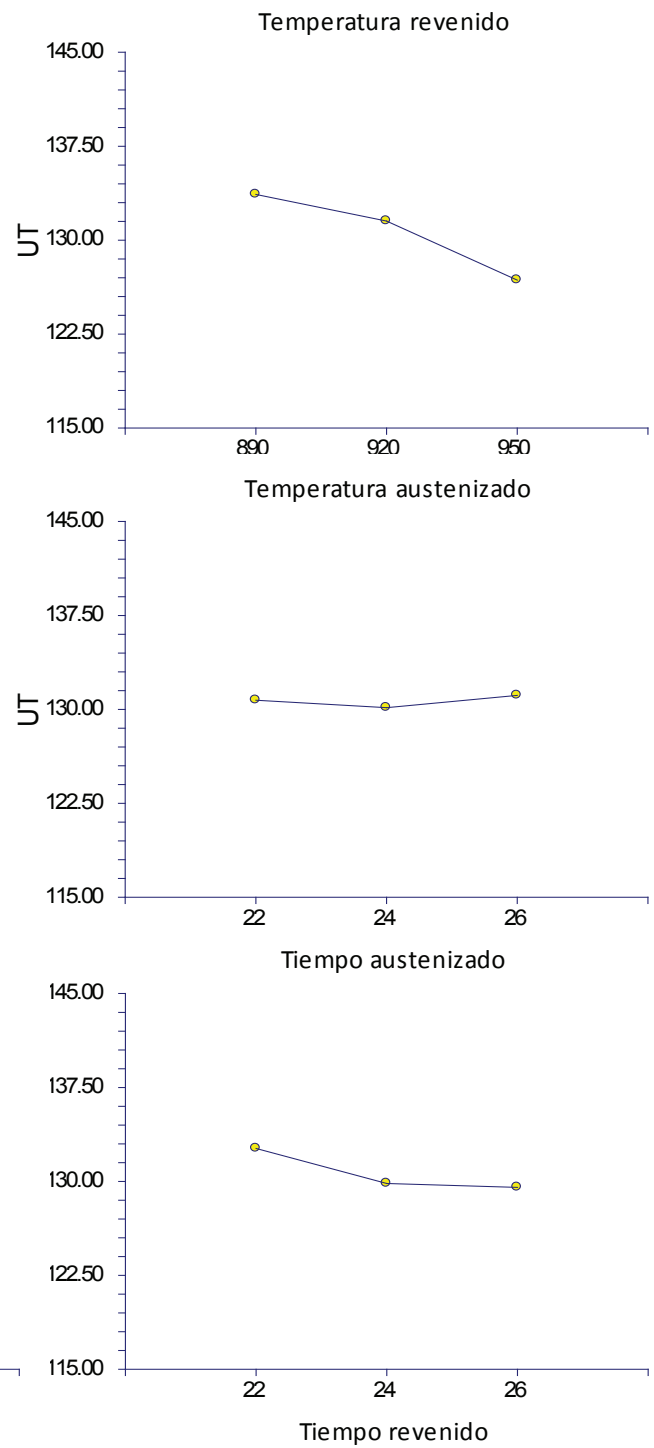

Figura 1. Efecto de los principales factores y sus niveles sobre el límite elástico (LE) (figuras lado izquierdo), y último esfuerzo a la tensión (UT) (figuras lado derecho) 

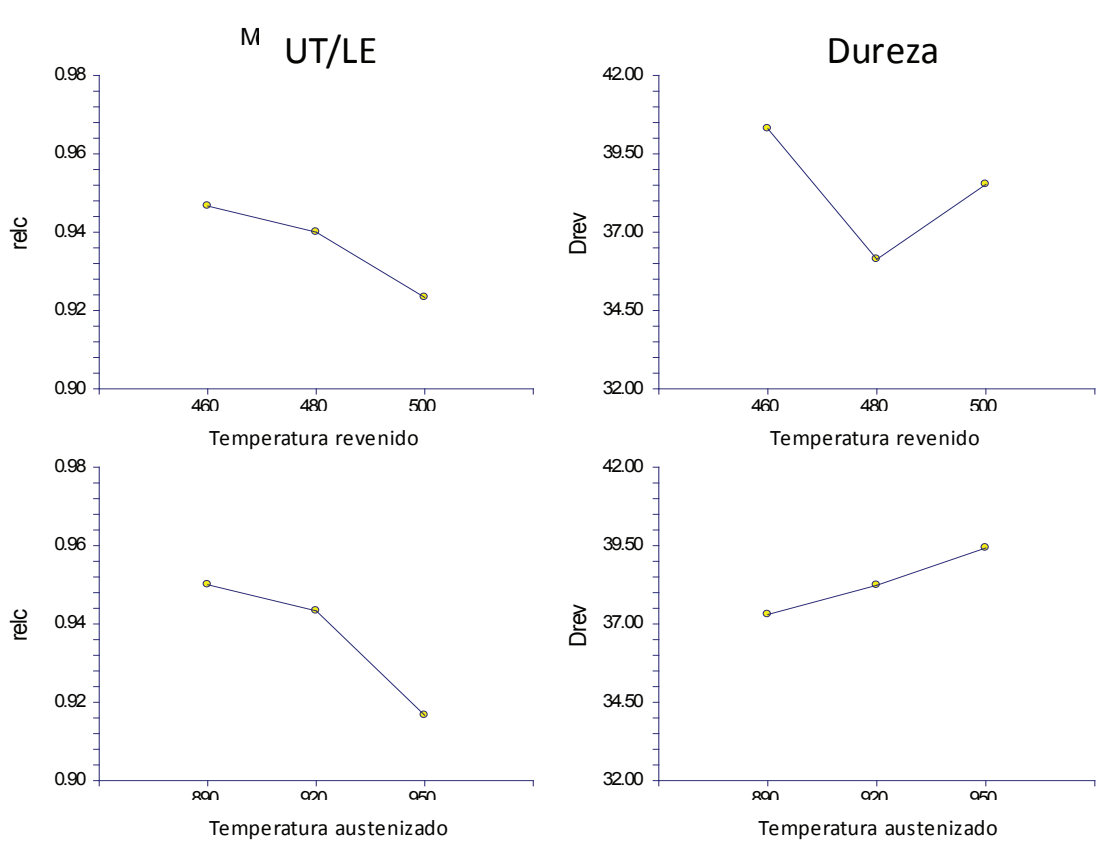

Figura 2. Efecto de los principales factores y sus niveles sobre la relación UT/LE (figuras lado izquierdo), y Dureza (figuras lado derecho)
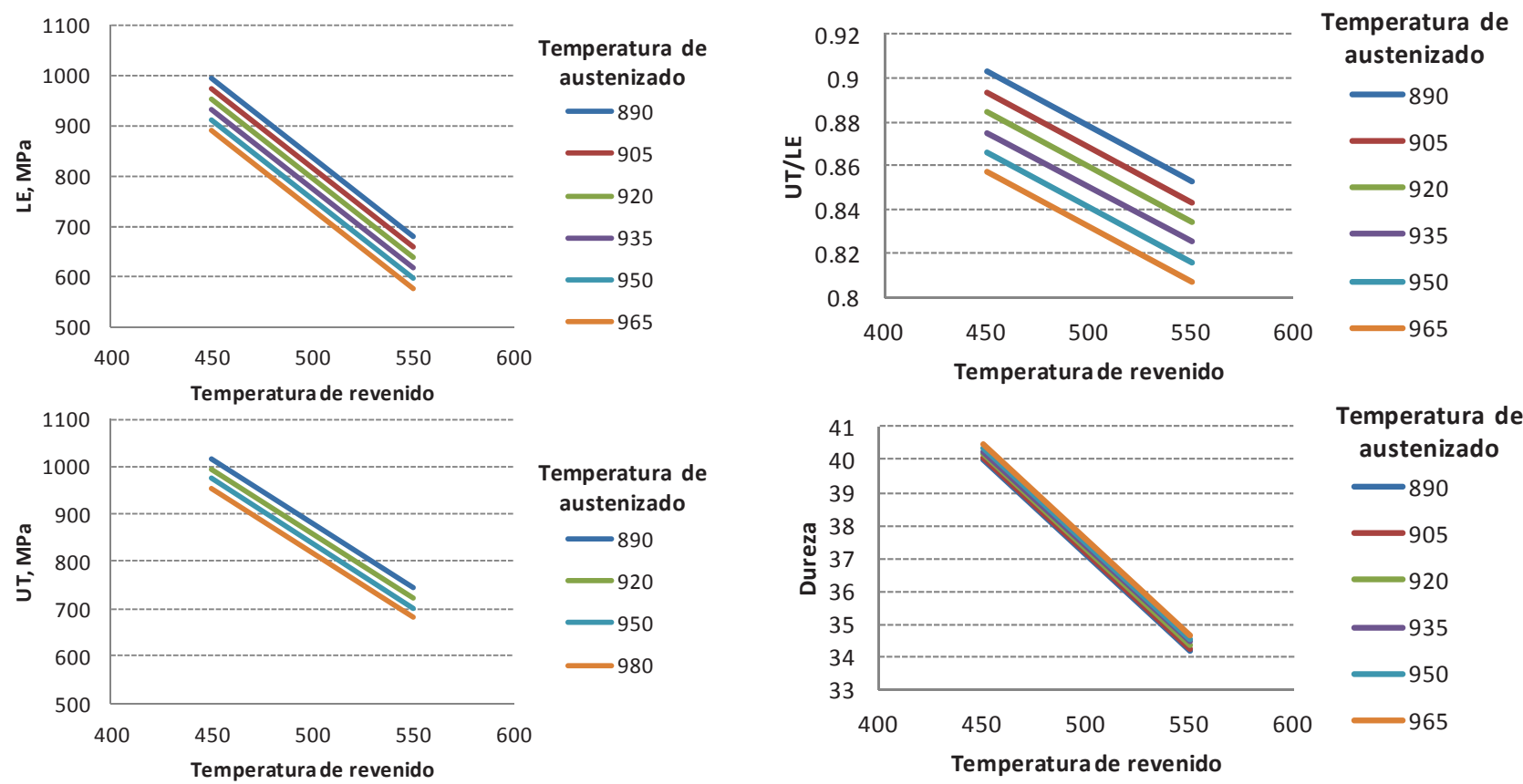

Figura 3. Curvas paramétricas para límite elástico (LE) (figura superior), y último esfuerzo a la tensión (UT) (figura intermedia superior), relación UT/LE (figura intermedia inferior) y Dureza (figura inferior), en función de las temperaturas de revenido y de austenizado 


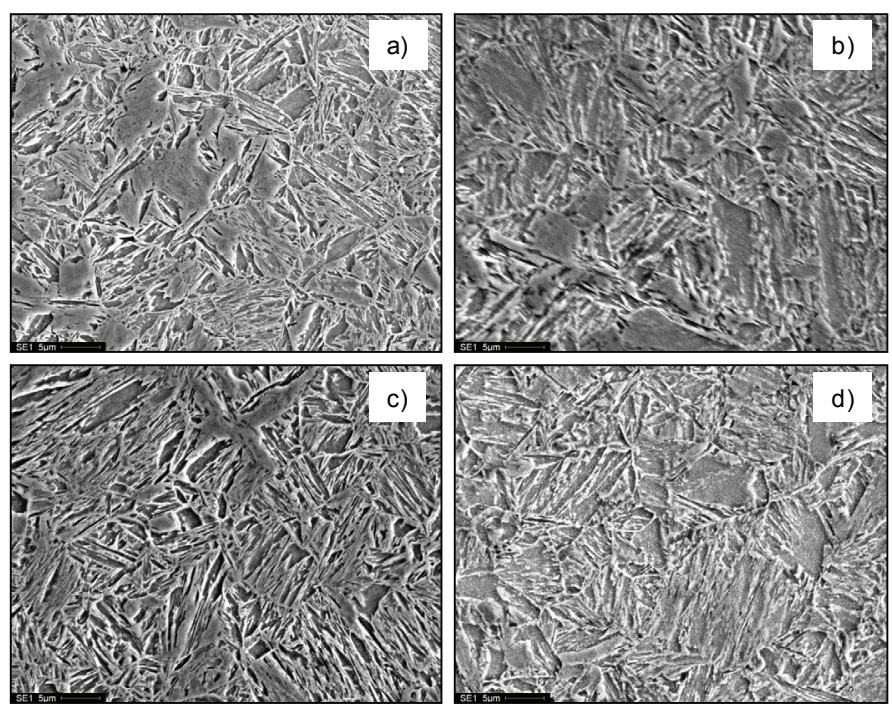

Figura 4. Fotomicrografías MEB de las muestras de tubo de la prueba 3 , a) austenizada, b) austenizada y revenida, y de la prueba 7, c) austenizada y d) austenizada y revenida mencionan Chung et al. (2012), no hay un cambio significativo en la microestructura ni en el tamaño de grano (todas las probetas analizadas presentaron un tamaño de grano entre 9.5 y 10 ASTM, $<5 \mu \mathrm{m}$ ). Por lo tanto, el tratamiento de revenido dado a estas probetas no afectaría las propiedades mecánicas, cumpliendo solo con el propósito de relevar los esfuerzos microestructurales. Esto puede corroborarse en el análisis de porcentajes de fases mostrado en la tabla 7.

La tabla 7 muestra que los porcentajes de fase (medido por conteo de fases en el analizador de imágenes) de algunas probetas seleccionadas (3, 4, 7 y 8) cambian ligeramente de la muestra austenizada a la austenizada y revenida, observándose una pequeña disminución de la fase austenítica (y aumento de la fase martensítica). Se puede observar que en el caso de la probeta 3, el porcentaje alcanzado de martensita durante el austenizado $\left(890^{\circ} \mathrm{C}\right)$ y temple, fue de $97.435 \%$, para luego aumentar después del revenido $\left(480^{\circ} \mathrm{C}\right)$ y enfriamiento al aire a $97.65 \%$, obteniendo valores de LE y UT por arriba de las especificaciones para este tipo de acero. En cambio, la probeta 8 presenta los valores más bajos de porcentaje de fase martensítica, tanto en el tratamiento de austenizado como en el austenizado y revenido. Este ciclo de tratamiento se realizó a las temperaturas más altas del diseño de pruebas (austenizado a $950^{\circ} \mathrm{C}$ y revenido a $500^{\circ} \mathrm{C}$ ), de donde se obtuvieron las más bajas propiedades mecánicas. Por lo tanto, las propiedades mecánicas registradas por los ciclos de tratamiento en las menores temperaturas de austenizado y de revenido se deben a que, bajo estas condiciones, es posible obtener una microestuctura martensítica con bajo porcentaje de fase austenítica. A menor temperatura de austenizado, me- nor será el tiempo de enfriamiento durante el templado con agua, incrementando la fase martensítica. Y a menor temperatura de revenido, es posible disminuir las tensiones residuales generadas por el templado, sin afectar significativamente la microestructura ni el porcentaje de fase martensítica, y manteniendo las propiedades mecánicas por arriba de los valores mínimos de la especificación 5CT J55.

Tabla 7. Análisis de fases de probetas provenientes de tubo tratadas térmicamente por austenizado y revenido

\begin{tabular}{llr}
\hline \multicolumn{1}{c}{ Prueba } & \multicolumn{1}{c}{ fases } & promedio \\
\hline \multirow{3}{*}{3 austenizado } & Martensita & 97.435 \\
& Austenita & 2.565 \\
\multirow{3}{*}{ austenizado y revenido } & Martensita & 97.655 \\
& Austenita & 2.345 \\
4 austenizado & Martensita & 96.89 \\
& Austenita & 3.03 \\
4 austenizado y revenido & Martensita & 97.11 \\
& Austenita & 2.62 \\
7 austenizado & Martensita & 96.53 \\
& Austenita & 3.24 \\
7 austenizado y revenido & Martensita & 97.4 \\
& Austenita & 2.6 \\
8 austenizado & Martensita & 95.925 \\
& Austenita & 4.075 \\
8 austenizado y revenido & Martensita & 95.98 \\
& Austenita & 4.016 \\
\hline
\end{tabular}




\section{Conclusiones}

El presente trabajo permite concluir lo siguiente:

1. Es posible aplicar un diseño experimental como el Taguchi L9 para encontrar, con un número muy pequeño de pruebas, el efecto de las variables del tratamiento térmico de austenizado y revenido sobre las propiedades mecánicas de un acero. En este caso, para muestras de tubo de acero API 5CT J55, la temperatura de revenido es el factor más importante para el logro de las propiedades mecánicas requeridas, seguido de la temperatura de austenizado.

2. A partir del análisis de varianza (ANOVA), se obtienen curvas paramétricas, que facilitan visualizar de manera gráfica y en forma inmediata las condiciones óptimas del tratamiento térmico. De la misma manera, a partir de una regresión múltiple de los resultados en propiedades mecánicas obtenidos del diseño experimental, es posible obtener ecuaciones empíricas que permiten encontrar, de manera sencilla y práctica, las condiciones óptimas de los tratamientos térmicos, dentro del rango experimental del estudio.

3. Los resultados de las propiedades mecánicas se explican mediante el estudio metalográfico de las muestras, ya que los tratamientos térmicos afectan la microestructura y el porcentaje de fases. Los resultados de este estudio indican que a una menor temperatura de revenido, principalmente, y a menor temperatura de austenizado, es posible tener mayor porcentaje de martensita y un tamaño de grano pequeño, lo cual se traduce en mejores propiedades mecánicas.

\section{Agradecimientos}

Los autores y la empresa Altos Hornos de México, S.A.B. de C.V. agradecen al CONACYT por el apoyo al Proyecto 200938 (Convocatoria 2012 del Programa de Estímulos a la Innovación).

\section{Referencias}

Andrews K.W. Empirical formulae for the calculation of some transformation temperatures. Journal of the Iron and Steel Institute, volumen 203, Parte 7, 1965: 721-727.

API specifications 5L. Specifications for line pipe, 43 ed., American Petroleum Institute, 2004.

Armentani E., Esposito R., Sepe R. The influence of thermal properties and preheating on residual stresses in welding. International Journal of Computational Materials Science and Surface Engineering, volumen 1, 2007: 146-162.
ASTM. Annual Book of ASTM Standards, 1984.

Barsoum Z. Residual stress analysis and fatigue of multi-pass welded tubular structures. Engineering Failure Analysis, volumen 15, 2008: 863-874.

Burgman P.M. Design of experiments the Taguchi way. Manufacturing Engineering, volumen 5, 1985: 44-46.

Chung P.C., Hama Y., Kim S., Lim J., Lee C. Effects of post-weld heat treatment cycles on microstructure and mechanical properties of electric resistance welded pipe welds. Materials and Design, volumen 34, 2012: 685-690.

Francis J.A., Cantin G.M.D., Mazur W., Bhadeshia H.K. Effects of weld preheat temperature and heat input on type IV failure. Science and Technology of Welding and Joining, volumen 14 (número 5), 2009: 436.

Hashemi S.H., Mohammadyani D. Characterisation of weldment hardness, impact energy and microstructure in API X65 steel. International Journal of Pressure Vessels and Piping, volumen 98, 2012: 8-15.

Hwang B., Kim Y.M., Lee S., Kim N.J., Ahn S.S. Correlation of microstructure and fracture properties of API X70 pipeline steels. Metallurgical and Materials Transactions A., volumen 36, 2005: 725-39.

Hwang B., Kim Y.G., Lee S., Kim Y.M., Kim N.J., Yoo J.Y., Effective grain size and charpy impact properties of high-toughness X70 pipeline steels. Metallurgical and Materials Transactions A., volumen 36, 2005: 2107-14.

Junhua K., Lin Z., Bin G., Pinghe L., Aihua W., Changsheng X. Influence of Mo content on microstructure and mechanical properties of high strength pipeline steel. Materials and Design, volumen 25, 2004: 723-8.

Kishor P. y Kolhe C.K. Prediction of microstructure and mechanical properties of multipass SAW. Journal of Materials Processing Technology, volumen 97, 2008: 241-9.

Kumar B.S., Tewari S.P., Prakash J. A review on effect of preheating and/or post weld heat treatmemt (PWHT) on mechanical behavior of ferrous metals. International Journal of Engineering Science and Technology, volumen 2 (número 4), 2010: 625-631.

Majta J. y Muszka K. Mechanical properties of ultra fine-grained HSLA and Ti-IF steels. Mater Sci Eng A, volumen 464, 2007: 186-91.

Noor A.S., Khan Z., Goel P., Kumar M., Agarwal G., Khan N.Z. Optimization of deep drilling process parameters of AISI 321 steel using Taguchi method. Procedia Materials Science, volumen 6, 2014: 1217-1225.

Ross P.J. Taguchi Techniques for Quality Engineering, McGraw Hill Book Company, Nueva York, 1996.

Santos D.B., Bruzszek R.K., Rodrigues P.C.M., Pereloma E.V. Formation of ultra-fine ferrite microstructure in warm rolled and annealed C-Mn steel. Mater Sci Eng A., volumen 346, 2003: 189-195.

Saini V.K, Khan Z., Noor A. Optimization of wire electric discharge machining of composite material (Al6061/SICP) using Ta- 
guchi Method. International Journal of Mechanical and Production Engineering, volumen 2, 2013: 61-64.

Tutar M., Aydin H., Yuce C., Yavuz N., Bayram A. The optimization of process parameters for friction stir spot-welded AA3003-H12 aluminum alloy using a Taguchi orthogonal array. Materials and Design, volumen 63, 2014: 789-797.

\section{Este artículo se cita:}

\section{Citación estilo Chicago}

Soria-Aguilar, Ma. de Jesús, Facundo Reyes-Guzmán, Francisco Raúl Carrillo-Pedroza, Fernando García-Garza, Humberto ÁlvarezJiménez, Luis Antonio Silva-Guajardo. Efecto del tratamiento térmico sobre las propiedades mecánicas y microestructura de un acero para tubería API 5CT J55. Ingeniería Investigación y Tecnología, XVI, 04 (2015): 539-550.

\section{Citación estilo ISO 690}

Soria-Aguilar M.J., Reyes-Guzmán F., Carrillo-Pedroza F.R., GarcíaGarza F., Álvarez-Jiménez H., Silva-Guajardo L.A. Efecto del tratamiento térmico sobre las propiedades mecánicas y microestructura de un acero para tubería API 5CT J55. Ingeniería Investigación y Tecnología, volumen XVI (número 4), octubre-diciembre 2015: 539-550.

\section{Semblanzas de los autores}

Ma. de Jesús Soria-Aguilar. Ingeniera Química Metalurgista de la Universidad Autónoma de Coahuila (UA$\mathrm{deC}$ ); es maestra en ciencias en metalurgia no ferrosa (1994) y doctora en ciencias en ingeniería metalúrgica (2005) por el Centro de Investigación y Estudios Avanzados del IPN, Unidad Saltillo. Actualmente, es catedrática investigadora de la Facultad de Metalurgia de la UAdeC desde 1999 e investigadora nivel I del sistema nacional de investigadores (SNI-CONACYT, México). Sus áreas de interés son el análisis, diseño y optimización de procesos metalúrgicos y comportamiento mecánico de los materiales.

Facundo Javier Reyes-Guzmán. Ingeniero mecánico con maestría en ingeniería metalúrgica y materiales. Se desempeña como ingeniero de productos en el Departamento de Ingeniería Metalúrgica y Servicio Técnico-Productos en Caliente de Altos Hornos de México S.A.B. de C.V. (AHMSA).

Francisco Raúl Carrillo-Pedroza. Ingeniero metalúrgico egresado del Instituto Tecnológico de Chihuahua con maestría en ciencias en metalurgia no ferrosa (1994) y doctorado en ciencias en ingeniería metalúrgica (2000) por el Centro de Investigaciones y de Estudios Avanzados del IPN (CINVESTAV-IPN) Unidad Saltillo (Coahuila). Desde el 2000 se desempeña como profesor-investigador en la Facultad de Metalurgia de la UAdeC. Es investigador nivel I del Sistema Nacional de Investigadores (SNI-CONACYT, México). Sus áreas de interés son el análisis de datos de procesos metalúrgicos y la metalurgia extractiva.

Fernando García-Garza. Es ingeniero metalurgista del Superintendente de Ingeniería Metalúrgica productos en Caliente, Decapada y No Planos del Departamento de Ingeniería Metalúrgica y Servicio Técnico, con 25 años de experiencia en Altos Hornos de México S.A.B. de C.V. (AHMSA).

Humberto Álvarez-Jiménez. Es licenciado en ciencias químicas por la Facultad de Ciencias Químicas de la UAdeC. Cuenta con la maestría en metalurgia con acentuación en siderurgia por la Facultad de Metalurgia, UAdeC. Actualmente es jefe de pruebas físicas y certificación del Laboratorio Central de AHMSA.

Luis Antonio Silva-Guajardo. Licenciado en administración de empresas y en contaduría pública, cuenta con estudios de maestría en administración y finanzas, actualmente cursa el doctorado en administración y alta dirección. Coordinador de vinculación y gestión tecnológica dentro de la Gerencia General de Sistemas de Calidad, Capacitación y Seguridad Industrial de AHMSAB, con 12 años de experiencia. Es responsable ante el CONACYT del Área de innovación y Desarrollo Tecnológico de AHMSA. 